\title{
ALLOWANCE FOR THE AGE OF CLAIMS \\ IN BONUS-MALUS SYSTEMS*
}

\author{
BY \\ Jean Pinquet ${ }^{1}$, Montserrat Guillén ${ }^{2}$, Catalina Bolancé2 $^{2}$
}

\begin{abstract}
The purpose of the paper is to use the age of claims in the prediction of risks. A dynamic random effects model on longitudinal count data is presented, and estimated on the portfolio of a major Spanish insurance company. The estimated autocorrelation coefficients of stationary random effects are decreasing. A consequence is that the predictive ability of a claim decreases with the lag between the period of risk prediction and the period of occurrence. There is a wide gap between the long term properties of actuarial and real-world experience rating schemes. This gap can be partly filled if the age of claims is taken into account in the actuarial model.
\end{abstract}

\section{KEYWORDS}

Time-independent and dynamic random effects. Autocorrelation function for stationary random effects.

\section{INTRODUCTION}

The purpose of the paper is to use the age of claims in the prediction of risks. This issue has already been addressed in the actuarial literature. Solutions are obtained from credibility models which can be updated (Gerber, Jones (1975)), and from credibility estimators with geometric weights (Sundt (1988)).

The rating models presented in this paper are obtained after statistical inference on longitudinal count data. Let us first clarify the reasons which lead to question the assumption of time-independence for the random effects.

\footnotetext{
* Pinquet acknowledges financial support from the Fédération Française des Sociétés d'Assurance. Guillén and Bolancé thank the Spanish CICYT grant SEC99-0693. We thank a referee for his comments.

1 (Corresponding author) University Paris X.

2 University of Barcelona.
} 
Hidden features of risk distributions vary with time, as do rating factors. The random effects added in an a priori rating model on longitudinal data should then be dynamic. Variations of rating factors between two dates should increase with the related lag, and the same result is expected for hidden features in risk distributions. Hence the predictive ability of a claim should decrease with the lag between the period of risk prediction and the period of occurrence. A stationary process for the random effects will relate the predictive power of claims to this lag. If the preceding intuition is verified, the estimated autocorrelation function of the random effects should be decreasing, a point already mentioned by Sundt (1988).

In Section 2, we present different Poisson models with random effects.

The variance of a time-independent random effect can be estimated from disaggregated data or from numbers of claims and frequency premiums which are summed across the periods. If the estimated variance obtained from disaggregated data is greater than the second one, the estimation of distributions for dynamic random effects can be considered. This condition is verified on our data set, which is drawn from the portfolio of a major Spanish insurance company.

An unconstrained autocorrelation function for dynamic random effects is then estimated from a Poisson model with regression components. For each lag, the corresponding autocorrelation is estimated from paired off products of lagged number-residuals and frequency-premiums. The autocorrelation function obtained in the empirical study decreases, but more slowly than a geometric one.

Optimal bonus-malus systems designed from a linear credibility approach are presented in Section 3 from the random effects models developed in Section 2.

In Section 4, we assess the consequences of a varying autocorrelation specification for the random effects on the dynamic of bonus-malus coefficients. An optimal bonus-malus system (later referred to as BMS) designed from a model with dynamic random effects and a decreasing autocorrelation function will behave in the following way. The no-claim discounts induced by a claimless year are lower than those obtained from the usual credibility model for a policyholder with a faultless history, but they are higher if claims were reported recently. The explanation is the same in both cases. The credibility granted to a given period of the past decreases rapidly as time goes by, due to the increase of risk exposure but mostly to the decrease in the autocorrelation coefficients.

Actuarial and real-world BMS differ with respect to the dynamic of bonusmalus coefficients. For example, the duration of a claimless history needed to offset the malus induced by a claim at fault is longer for actuarial than for real-life BMS. The aforementioned properties of the BMS with dynamic random effects allow us to reduce this difference.

Increases in premiums induced by claims from the different BMS are not very different in the empirical study. On the whole, an optimal BMS derived from a Poisson model with dynamic random effects seems acceptable to policyholders, if the estimated correlogram is decreasing. Besides, it would 
entail strong incentives to careful driving for the drivers who reported a claim recently.

Another difference is that an optimal BMS designed from rating models with dynamic random effects reaches its limit faster than the usual ones. Besides, total credibility does not converge towards one, which entails a lower dispersion for the bonus-malus coefficients. In real-life situations, the dispersion of bonus-malus coefficients is much lower than what is obtained from actuarial models. Allowing for the age of claims in an optimal BMS reduces the dispersion of the bonus-malus coefficients.

Finally, the applicability of the results obtained in the paper is briefly discussed in Section 5. A possibly useful result for practitioners is the following. An optimal BMS estimated from short histories and applied to a longer duration will overestimate the individual credibilities. This result occurs if the autocorrelation function of the random effects decreases with the lag. Hence, attention should be paid to the link between the length of the histories used in risk assessment and the duration of application of the BMS.

\section{MOMENT-BASED ESTIMATORS FOR LONGITUDINAL COUNT DATA}

\subsection{Two estimators for the variance of a time-independent random effect}

Let us consider a portfolio composed of $p$ policyholders. If we have an unbalanced panel data set, the policyholder $i$ is observed during $T_{i}$ periods, with $1 \leq T_{i} \leq T_{\max }$. If $n_{i, t}$ is the number of claims reported by the policyholder $i$ in period $t$, the distributions retained for the frequency risk model are mixtures of Poisson distributions. Their likelihood is equal to

$$
L\left(n_{i, t}\right)_{1 \leq t \leq T_{i}}=E\left[\prod_{t=1}^{T_{i}} P_{\lambda_{i, t} U_{i}}\left(n_{i, t}\right)\right] \text {, with } P_{\lambda}(n)=\exp (-\lambda) \frac{\lambda^{n}}{n !} .
$$

The expectation is taken with respect to the random effect $U_{i}$, and the coefficients $\lambda_{i, t}$ depend on regression components (represented by a line-vector $x_{i, t}$ ) and on the duration of the period $d_{i, t}$. We write

$$
\lambda_{i, t}=d_{i, t} \exp \left(x_{i, t} a\right) ; a \in \mathbb{R}^{k},
$$

where $a$ is a column-vector of parameters and where $k$ is the number of regression components. The random effects $\left(U_{i}\right)_{i=1, \ldots, p}$ are assumed i.i.d., and the two first moments are

$$
E\left(U_{i}\right)=1 ; V\left(U_{i}\right)=\sigma_{U}^{2} .
$$

Within a semiparametric approach, we do not completely specify the distributions of the random effects. Estimators and predictors are obtained from second order moments of the random effects and from the maximum likelihood estimation of the Poisson model with regression components and 
without random effects. Let us denote the cumulated number of claims and frequency risk for the policyholder $i$ as $n_{i}=\sum_{t=1}^{T_{i}} n_{i, t}$ and $\lambda_{i}=\sum_{t=1}^{T_{i}} \lambda_{i, t}$. Two consistent estimators of $\sigma_{U}^{2}$ can be retained, which are

$$
\widehat{\sigma_{U}^{2}}{ }^{1}=\frac{\sum_{i, t}\left[\left(n_{i, t}-\hat{\lambda}_{i, t}\right)^{2}-n_{i, t}\right]}{\sum_{i, t} \hat{\lambda}_{i, t}^{2}} ;{\widehat{\sigma_{U}^{2}}}^{2}=\frac{\sum_{i}\left(n_{i}-\hat{\lambda}_{i}\right)^{2}-n_{i}}{\sum_{i} \hat{\lambda}_{i}^{2}},
$$

where $\hat{\lambda}_{i, t}$ and $\hat{\lambda}_{i}$ are the frequency-premiums computed from likelihood maximization in the Poisson model without random effects. These two estimators are unbiased, and the second estimator should be preferred because its variance is lower. The intuition is that this estimator uses more information. Besides, ${\widehat{\sigma_{U}^{2}}}^{1}$ reflects a short term predictive ability of the claims. It should be greater than an estimator based on longer durations if the predictive ability of claims decreases with their age. The inequality

$$
0<{\widehat{\sigma_{U}^{2}}}^{2}<{\widehat{\sigma_{U}^{2}}}^{1}
$$

is fulfilled on our data set (see Section 4). It is a necessary condition for the estimation of simple dynamic random effects specifications (see Pinquet, Guillén, Bolancé (2000)).

\subsection{A Poisson model with dynamic random effects}

We now replace the time-independent random effect $U_{i}$ by a dynamic random effect $U_{i, t}$. The likelihood of the random effects model is equal to

$$
L\left(n_{i, t}\right)_{1 \leq t \leq T_{i}}=E\left[\prod_{t=1}^{T_{i}} P_{\lambda_{i, t}} U_{i, t}\left(n_{i, t}\right)\right] .
$$

As mentioned in the introduction, the time-dependence assumption for the random effects is natural. Two assumptions are retained on the random effects, which are the following.

- The distribution of $\underset{1 \leq t \leq T_{i}}{v e c}\left(U_{i, t}\right)$ depends only on $T_{i}$.

- If the distribution of $\underset{1 \leq t \leq T_{i}}{\operatorname{vec}}\left(U_{i, t}\right)$ is that of $\underset{1 \leq t \leq T_{i}}{\operatorname{vec}}\left(U_{t}\right)$ the distribution of $\underset{1 \leq t \leq T_{\max }}{v e c}\left(U_{t}\right)$ is supposed to be stationary. This invariance assumption with respect to time translations means that the predictive ability of a claim will depend on the lag between the period of risk prediction and the period of occurrence. We suppose that the squared random effects are integrable. 
The covariances and autocorrelation coefficients for the random effects are denoted as

$$
\operatorname{Cov}\left(U_{t}, U_{t-h}\right)=\rho_{U}(h) \sigma_{U}^{2} ; 0 \leq h<t \leq T_{\max },
$$

with: $-1 \leq \rho_{U}(h) \leq 1 ; \rho_{U}(0)=1$.

From the moment equations derived in the random effects model

$$
\begin{aligned}
& E\left[\sum_{i, t}\left(N_{i, t}-\lambda_{i, t}\right)^{t} x_{i, t}\right]=0\left(0 \in \mathbb{R}^{k}\right) ; \\
& E\left[\sum_{i, t}\left(N_{i, t}-\lambda_{i, t}\right)^{2}-N_{i, t}-\lambda_{i, t}^{2} \sigma_{U}^{2}\right]=0 ; \\
& E\left[\sum_{i \mid T_{i}>h} \sum_{T_{i} \geq t>h}\left(N_{i, t}-\lambda_{i, t}\right)\left(\begin{array}{c}
\left.\left(N_{i, t-h}-\lambda_{i, t-h}\right)-\lambda_{i, t} \lambda_{i, t-h} \sigma_{U}^{2} \rho_{U}(h)\right]=0, \\
\left(0<h<T_{\max }\right)
\end{array}\right]\right.
\end{aligned}
$$

we obtain consistent moment-based estimators for $a, \sigma_{U}^{2}$ and $\left(\rho_{U}(h)\right)_{0<h<T_{\max }}$ The empirical counterpart of the moment equation related to $a$ leads to

$$
\sum_{i, t}\left(n_{i, t}-\hat{\lambda}_{i, t}\right)^{t} x_{i, t}=0, \hat{\lambda}_{i, t}=d_{i, t} \exp \left(x_{i, t} \hat{a}\right) .
$$

Hence, the maximum likelihood estimator of $a$ in the Poisson model without random effects (i.e. the a priori rating model) is a consistent estimator of $a$ in the model with random effects. From the second moment equation, a consistent estimator of $\sigma_{U}^{2}$ is $\widehat{\sigma_{U}^{2}}{ }^{1}$ (see (1)). Finally, the estimated correlogram of $U$ is obtained from

$$
\widehat{\sigma_{U}^{2}{ }^{1}} \rho_{U}(h)=\frac{\sum_{i \mid T_{i}>h} \sum_{T_{i} \geq t>h}\left(n_{i, t}-\hat{\lambda}_{i, t}\right)\left(n_{i, t-h}-\hat{\lambda}_{i, t-h}\right)}{\sum_{i \mid T_{i}>h} \sum_{T_{i} \geq t>h} \hat{\lambda}_{i, t} \hat{\lambda}_{i, t-h}}
$$

for $0<h<T_{\max }$. All these estimators are consistent and asymptotically normal. They are given in Zeger (1988), along with modified estimators which use weights related to overdispersion and autocorrelation in the regression in order to reduce the asymptotic variance.

\section{LINEAR CREDIBILITY PREDICTORS DERIVED FROM THE PRECEDING MODELS}

Let $\left(n_{t}\right)_{1 \leq t \leq T \leq T_{\max }}$ be the history of claims recorded on an insurance contract (we suppress the individual index in order to simplify the notations). A linear credibility predictor (Bühlmann (1967)) for period $T+1$ is obtained from a 
regression derived in the model with random effects. The predictor is equal to $\widehat{a}+\sum_{t=1}^{T} \widehat{b}_{t} n_{t}$, with

$$
\left(\hat{a}, \widehat{b}_{1}, \ldots, \widehat{b}_{T}\right)=\arg \min _{a,\left(b_{t} t_{t=1} \ldots, T\right.} \widehat{E}\left[\left(U_{T+1}-a-\sum_{t=1}^{T} b_{t} N_{t}\right)^{2}\right],
$$

where the expectation is estimated in the random effects model.

A linear predictor of the type $\widehat{a}+\widehat{b}\left(\sum_{t=1}^{T} n_{t}\right)$ is obtained from an expected value principle with the negative binomial model (see Lemaire (1985), and Dionne, Vanasse (1989)). The purpose of the paper is to obtain time-dependent coefficients in the linear combination. The intuition is that $b_{t}$ should decrease with the age of the period $t$.

Since $E\left(U_{T+1}\right)=1$, we have $\widehat{a}+\sum_{t=1}^{T} \widehat{b}_{t} \widehat{E}\left(N_{t}\right)=1$. Now $\widehat{\lambda}_{t}$, the frequency premium derived from likelihood maximization in the a priori rating model, converges towards the frequency risk $E\left(N_{t}\right)$ computed in the model with random effects (see the comments following equation (3)). Then we have

$$
\begin{gathered}
\widehat{a}+\sum_{t=1}^{T} \widehat{b}_{t} n_{t}=1+\sum_{t=1}^{T} \widehat{b}_{t}\left(n_{t}-\widehat{\lambda}_{t}\right), \text { with } \\
\left(\widehat{b}_{t}\right)_{t=1, \ldots, T}=\arg \min _{\left(b_{t}\right)_{t=1, \ldots, T}} \widehat{V}\left[\left(U_{T+1}-\sum_{t=1}^{T} b_{t} N_{t}\right)^{2}\right]=[\widehat{V}(N)]^{-1} \widehat{\operatorname{Cov}}\left(N, U_{T+1}\right) .
\end{gathered}
$$

We write $N=\underset{1 \leq t \leq T}{v e c}\left(N_{t}\right)$. From the consistent estimators given in Section 2.2, the estimators of the individual moments of interest are

$$
\begin{gathered}
\widehat{V}\left(N_{t}\right)=\widehat{\lambda_{t}}+\widehat{\sigma_{U}^{2}} \hat{\lambda}_{t}^{2} ; \widehat{\operatorname{Cov}}\left(N_{t}, N_{t^{\prime}}\right)=\hat{\lambda}_{t} \hat{\lambda}_{t^{\prime}},{\widehat{\sigma_{U}^{2}}}^{1} \widehat{\rho}_{U}\left(\left|t-t^{\prime}\right|\right)\left(t \neq t^{\prime}\right) ; \\
\widehat{\operatorname{Cov}}\left(N_{t}, U_{T+1}\right)=\hat{\lambda_{t}}{\widehat{\sigma_{U}^{2}}}^{1} \rho_{U}(T+1-t) .
\end{gathered}
$$

The bonus-malus coefficient can be written as

$$
\left(1-\sum_{i=1}^{T} \text { cred }_{t}\right)+\sum_{t=1}^{T} \operatorname{cred}_{t} \frac{n_{t}}{\hat{\lambda}_{t}}
$$

where $\left(\text { cred }_{t}=\hat{b}_{t} \hat{\lambda}_{t}\right)_{t=1, \ldots, T}$ are the credibility coefficients, which are the solutions of the linear system with $t=1, \ldots, T$ equations

$$
\left(1+\hat{\lambda}_{t}{\widehat{\sigma_{U}^{2}}}^{1}\right) \operatorname{cred}_{t}+\hat{\lambda}_{t} \sum_{t^{\prime} \neq t}{\widehat{\sigma_{U}^{2}}}^{1} \hat{\rho}_{U}\left(\left|t-t^{\prime}\right|\right) \operatorname{cred}_{t^{\prime}}=\widehat{\lambda}_{t}{\widehat{\sigma_{U}^{2}}}^{1} \hat{\rho}_{U}(T+1-t)
$$

This linear credibility system can be used with the unconstrained correlogram estimated in Section 2.2. 
Properties of the credibility coefficients derived from equation (5) are not simple to obtain in a general setting. If the frequency premiums are negligible with respect to one, we infer from this equation

$$
\operatorname{cred}_{t} \sim \hat{\lambda}_{t}{\widehat{\sigma_{U}}}^{1} \hat{\rho}_{U}(T+1-t)
$$

A sequence of credibility coefficients should have the same shape as that of a correlogram with the same length and a reversed index.

Total credibility does not converge to one when $T$ goes to infinity if the autocorrelation function decreases rapidly, and the limit can be very inferior to one. Let us consider for example a Gaussian $A R(1)$ process for the additive random effects $W_{t}=\log \left(U_{t}\right)$. With our data base, we obtain $\hat{\rho} w(h)=0.79^{h}$ (see Pinquet, Guillén, Bolancé (2000) for details related to the estimation). The total credibility for an average risk $\left(\widehat{\lambda}_{t}=0.09 \forall t\right)$ converges towards 0.214 when $T$ converges towards infinity. This limit is obtained in round figures after twenty years. It corresponds to the maximum bonus applied to the a priori frequency premium of a policyholder with a claimless history.

Simple updating formulas do not seem to be available for the credibility coefficients. Gerber and Jones (1975) prove that linear updating formulas exist under conditions which differ from the stationarity assumption retained in this paper for the random effects.

\section{EMPIRICAL RESULTS}

\subsection{The data set}

The working sample represents ten per cent of the portfolio of a major Spanish insurance company. We selected only policies covering cars for private use. The durations of individual histories range from one to seven years, hence $T_{\max }=7$ with the notations of the paper. Policyholders were observed between 1991 and 1997, and indicators of the calendar years are part of the regression components in order to allow for a trend in the past (see Besson, Partrat (1992) for optimal BMS with a trend). The other rating factors retained in the regression are the gender, the geographical area, the age of the driving licence, the seniority and age of the policyholder, the coverage level and the power of the vehicle.

In order to have similar rates of arrival and attrition in the working sample and in the portfolio, we selected the policyholders in the following way. Ten per cent of the policyholders present in 1991 were selected at random, and kept in the working sample as long as possible. Ten per cent of the newcomers in 1992 were included in the working sample, and so on. The size of the working sample increases from 120000 in 1991 to 200000 in 1997 (in round figures). The attrition rate varies between $8.5 \%$ and $10 \%$. The working sample is an unbalanced panel data set which is composed of 269388 policyholders and of 1172701 periods. The average frequency of claims at fault per year is equal to 0.09 . All the period durations are equal to one year, which means 
that the characteristics of the policyholders are known only at each anniversary date. This inaccuracy in the observation of the regression components is of no consequence in our opinion.

\subsection{Estimators for the correlogram of the random effects}

The two consistent estimators quoted in Section 2.1 for the variance of a timeindependent random effect are respectively

$$
\begin{gathered}
\widehat{\sigma_{U}^{2}}{ }^{1}=\frac{\sum_{i, t}\left(n_{i, t}-\hat{\lambda}_{i, t}\right)^{2}-n_{i, t}}{\sum_{i, t} \widehat{\lambda}_{i, t}^{2}}=\frac{118554.78-105655}{10167.12}=1.269 . \\
{\widehat{\sigma_{U}^{2}}}^{2}=\frac{\sum_{i}\left(n_{i}-\widehat{\lambda}_{i}\right)^{2}-n_{i}}{\sum_{i} \widehat{\lambda}_{i}^{2}}=\frac{144879.33-105655}{50359.14}=0.779 .
\end{gathered}
$$

As expected in Section 2.1, we have ${\widehat{\sigma_{U}^{2}}}^{2}<{\widehat{\sigma_{U}^{2}}}^{1}$.

The correlogram of $\left(U_{t}\right)_{1 \leq t \leq 6}$ is estimated from equations (1) and (4). We obtain

TABLE 1

AUTOCORRELATION COEFFICIENTS

\begin{tabular}{lcccccc}
\hline \hline$h$ (lag) & 1 & 2 & 3 & 4 & 5 & 6 \\
\hline$\widehat{\rho}_{U}(h)$ & 0.632 & 0.485 & 0.462 & 0.436 & 0.360 & 0.348 \\
\hline \hline
\end{tabular}

The difference between $\widehat{\rho}_{U}(1)$ and $\rho_{U}(0)=1$ reflects the loss of predictive ability related to a claim after one year. Owing to the shape of the correlogram, the predictive ability of claims decreases with their age. The consequences are assessed in the following section.

The moment-based estimators retained in this paper are unconstrained. For instance, the estimated autocorrelation coefficients are not bound to belong to $[-1,1]$. This constraint is fulfilled by the preceding estimators, and a multivariate log-normal distribution for the $\left(U_{t}\right)_{1 \leq t \leq 6}$ can be matched with the values of Table 1 (see Pinquet, Guillén, Bolancé (2000) for more details and further discussion in case of misspecification).

\subsection{Experience rating from the different models}

In the following tables, credibility coefficients for the different periods are computed for an insurance contract with an average frequency premium, which is equal to 0.09 per year. Within a linear credibility approach, we use the Poisson models with random effects presented in Section 2. The next table 
provides credibility coefficients computed from time-independent and dynamic random effects. The coefficients are computed for histories ranging from one to six years. We used the usual credibility formula for time-independent random effects, and the linear credibility system given in Section 3 for the other model. We estimated the variance of the time-independent random effect from the number of claims and frequency-premiums summed across the periods. Hence, we retained ${\widehat{\sigma_{U}^{2}}}^{2}=0.779$ instead of ${\widehat{\sigma_{U}^{2}}}^{1}=1.269$ (see equation (6)).

Remember that a credibility coefficient is a bonus if no claim is reported.

TABLE 2

CREDIBILITY COEFFICIENTS FOR AN AVERAGE RISK (PERCENTAGE) DYNAMIC VS. TIME-INDEPENDENT RANDOM EFFECTS

\begin{tabular}{lcccccccc}
\hline \hline $\begin{array}{c}\text { Duration } \\
\text { of histories }\end{array}$ & \multicolumn{4}{c}{$\begin{array}{c}\text { Credibility per year (\%) } \\
\text { dynamic random effects }\end{array}$} & \multicolumn{3}{c}{$\begin{array}{c}\text { Total credibility (\%) } \\
\text { dynamic }\end{array}$} \\
& $\mathrm{t}=1$ & $\mathrm{t}=2$ & $\mathrm{t}=3$ & $\mathrm{t}=4$ & $\mathrm{t}=5$ & $\mathrm{t}=6$ & time-independent \\
random effects & random effects \\
\hline 1 year & 6.47 & 0 & 0 & 0 & 0 & 0 & 6.47 & 6.55 \\
2 years & 4.57 & 6.17 & 0 & 0 & 0 & 0 & 10.74 & 12.29 \\
3 years & 4.15 & 4.32 & 5.98 & 0 & 0 & 0 & 14.45 & 17.37 \\
4 years & 3.74 & 3.94 & 4.14 & 5.83 & 0 & 0 & 17.65 & 21.89 \\
5 years & 2.83 & 3.57 & 3.82 & 4.03 & 5.72 & 0 & 19.97 & 25.95 \\
6 years & 2.66 & 2.68 & 3.46 & 3.71 & 3.94 & 5.65 & 22.10 & 29.60 \\
\hline \hline
\end{tabular}

For a given duration of the individual history, the credibility coefficients of the last table decrease with the lag between the prediction period and the current period, as do the autocorrelation coefficients. For example, the credibility given in Table 2 for the last year of a six years history outweighs the credibility of the two first years. Besides, total credibility (and hence the bonus applied to a claimless history) is lower if dynamic random effects are used in the rating model.

Let us now perform an impulse-response analysis of the evolution of the bonus-malus coefficient if one claim is reported during the first year, and none during the years that follow. We compare the two BMS for a car with the average frequency premium. Bonus-malus coefficients are expressed as percentages.

TABLE 3

IMPULSE-RESPONSE ANALYSIS OF BONUS-COEFFICIENTS AFTER ONE CLAIM

\begin{tabular}{lcccccc}
\hline \hline Years & 1 & 2 & 3 & 4 & 5 & 6 \\
\hline time-independent random effects & 166.2 & 156 & 147 & 139 & 131.7 & 125.2 \\
dynamic random effects & 165.5 & 140 & 131.7 & 123.8 & 111.4 & 107.5 \\
\hline \hline
\end{tabular}


The no-claim discounts are higher for the rating model with dynamic random effects. For instance, the discounts from period 1 to period 2 are equal to 6 and 15 per cent in the two models. This important difference is due to the fact that claimless periods increase risk exposure but also the age of claims reported in the past. This property of the rating model with dynamic random effects can be related to some clauses found in real-life BMS, which provide important discounts for bad drivers with recent good behaviour. In France for instance, a driver with a bonus-malus coefficient greater than the coefficient applied to beginners (less than three percent of the drivers are concerned) is rated according to this coefficient after two consecutive claimless years. This feature of real-life or optimal BMS entails strong incentives to drive carefully for policyholders with a bad accident record. Notice that economic analysis suggests that optimal insurance contracts with moral hazard should penalize recent claims more than older ones (Henriet, Rochet (1986)).

As shown in Table 3, the duration of a claimless history needed to offset the malus induced by a claim at fault is longer for an actuarial BMS designed from time-independent random effects than for the other BMS. If the frequency premium per year is equal to 0.09 , ten claimless years are needed to offset the malus with the usual BMS. On the other hand, five claimless years are almost enough to obtain the same result if the other BMS is used. This duration is closer to those derived from real-life BMS, which range from three to five years in most cases (see Lemaire (1995) for a thorough description of compulsory BMS).

Let us compare total credibility for longer durations. We need to extend the correlogram for higher values of the lag. A possible extension is derived from the Yule-Walker equations applied to the additive random effects $W_{t}=$ $\log \left(U_{t}\right)$. If we assume that the past is summarized by the last six years for the random effects process, we obtain an $A R(6)$ specification for the additive random effects. In that case, a parametric specification is needed in order to link the second order moments of the $W_{t}$ with the corresponding moments of $U_{t}$. A multivariate Gaussian distribution for the additive random effects can be considered (see Pinquet, Guillén, Bolancé (2000) for more details). With an average risk and from the correlogram given in Table 1, we obtain

TABLE 4

LONG-TERM BEHAVIOUR OF TOTAL CREDIBILITY (PERCENTAGE)

\begin{tabular}{ccc}
\hline \hline $\begin{array}{c}\text { Duration of } \\
\text { histories }\end{array}$ & $\begin{array}{c}\text { time-independent } \\
\text { random effects }\end{array}$ & $\begin{array}{c}\text { dynamic } \\
\text { random effects }\end{array}$ \\
\hline 10 years & 41.2 & 27.7 \\
20 years & 58.4 & 32.6 \\
40 years & 73.7 & 34.1 \\
\hline \hline
\end{tabular}

The result obtained in the last column is striking. After almost a full life as a car driver, a policyholder with a claimless history obtains a frequency-bonus of only 34 per cent, which is about five times the bonus after the first year. 
In the last table, we compare the dispersion of the bonus-malus coefficients derived from the two models. We derive the standard deviation of bonusmalus coefficients for different durations of the histories. Computations are performed in the two different random effects models for an individual with an average frequency risk. The autocorrelation function of the dynamic random effects is extended as indicated before Table 4 . The variance of the bonusmalus coefficients are those of the linear regression which defines the linear credibility predictor, that is to say

$$
\widehat{{ }^{t} \operatorname{Cov}}\left(N, U_{T+1}\right)[\widehat{V}(N)]^{-1} \widehat{\operatorname{Cov}}\left(N, U_{T+1}\right)
$$

with the notations of Section 3. For different durations of the history, we obtain

TABLE 5

STANDARD DEVIATION OF BONUS-MALUS COEFFICIENTS

\begin{tabular}{lccccc}
\hline \hline duration of the history (years) & 1 & 5 & 10 & 20 & 40 \\
\hline time-independent random effects & 0.226 & 0.450 & 0.567 & 0.674 & 0.758 \\
dynamic random effects & 0.228 & 0.355 & 0.389 & 0.398 & 0.399 \\
\hline
\end{tabular}

In real-life situations, the dispersion of bonus-malus coefficients is much lower than what is obtained from actuarial models. For instance, the coefficient of variation of the bonus-malus coefficients in Belgium was equal to 0.154 in 1992 (see Lemaire (1995) for the distribution of bonus-malus coefficients at that time). The standard deviation of bonus-malus coefficients in an actuarial BMS is also the coefficient of variation, due to the fairness property of these systems. The differences between actuarial and real-life BMS are lower if the age of claims is allowed for in the statistical model.

\section{CONCLUding REMARKS}

The main features of an optimal BMS derived from stationary random effects with a decreasing correlogram seem acceptable to policyholders. The no-claim discounts are lower for claimless drivers than those derived from the usual optimal BMS. On the other hand, they can be much higher for policyholders who reported claims recently. Such systems would entail strong incentives for these drivers to drive carefully.

A useful result for the application of actuarial models is the following. If the autocorrelation between stationary random effects decreases with the lag, the variance of a time-independent random effect (estimated from aggregated numbers and frequency-premiums) will decrease with the average duration of the histories used in the estimation. For instance, the variance estimated from the observations of the first period is equal to 1.09 , whereas the variance obtained from the full histories is equal to ${\widehat{\sigma_{U}^{2}}}^{2}=0.78$ (see Section 4.2). In this 
framework, an optimal BMS estimated from short histories and applied to a longer duration will overestimate the individual credibilities. This result provides a supplementary reason to use the whole history of the policyholders in the statistical analysis.

\section{REFERENCES}

1. Besson, J.L. and PARTrat, C. (1992) Trend et systèmes de bonus-malus. ASTIN Bulletin 22, 11-32.

2. BüHlmann, H. (1967) Experience rating and credibility. ASTIN Bulletin 4, 199-207.

3. Dionne, G. and Vanasse, C. (1989) A generalization of automobile insurance rating models: the negative binomial distribution with a regression component. ASTIN Bulletin 19, 199-212.

4. GERBER, H. and JONES, D. (1975) Credibility formulas of the updating type. Transactions of the Society of Actuaries 27, 31-52.

5. Henriet, D. and Rochet, J.C. (1986) La logique des systèmes bonus-malus en assurance automobile. Annales d'Economie et de Statistiques, 133-152.

6. Lemaire, J. (1985) Automobile Insurance: Actuarial Models. Kluwer Academic Publishers.

7. Lemaire, J. (1995) Bonus-Malus Systems in Automobile Insurance. Kluwer Academic Publishers.

8. Pinquet, J., Guillen, M. and Bolance, C. (2000) Long-range contagion in automobile insurance data: Estimation and implications for experience rating. Working paper 2000-43, http://thema.u-paris 10. fr.

9. SUNDT, B. (1988) Credibility estimators with geometric weights. Insurance: Mathematics and Economics 7, 113-122.

10. ZEGER, L.S. (1988) A regression model for time series of counts. Biometrika 74, 721-729.

\section{JEAN PINQUeT}

U.F.R. de Sciences Economiques

Université de Paris $X$

200, avenue de la République

92001 Nanterre Cedex

France

e-mail:pinquet@u-paris10.fr

Montserrat Guillén

Departament d'Econometria, Estadística i Economia Espanyola

Universitat de Barcelona

Diagonal, 690

08034 Barcelona

Spain

e-mail: guillen@eco.ub.es

Catalina Bolancé

Departament d'Econometria, Estadística i Economia Espanyola

Universitat de Barcelona

Diagonal, 690

08034 Barcelona

Spain

e-mail:bolance@eco.ub.es 